Katharina Helbig ${ }^{1,2}$, Jiazhi Wang ${ }^{3}$, Jörg Bischof ${ }^{4}$, Doris Börner ${ }^{5}$, Stefan Dennenmoser ${ }^{2,6}$, Heinz G. Endres ${ }^{7}$, Johannes Fleckenstein ${ }^{8}$, Eva-Maria Helbig ${ }^{9}$, Hans-Udo Richarz ${ }^{2,10}$, Uwe Schütz ${ }^{11,12}$, Jaap van der Wal ${ }^{13}$, Werner Klingler ${ }^{1,2,14,15}$

${ }^{1}$ Klinik für Anästhesie, Intensivmedizin, Notfallmedizin und Schmerztherapie, SRH Kliniken Landkreis Sigmaringen GmbH, Sigmaringen, Deutschland

${ }^{2}$ Fascia Research Group, Sektion Experimentelle Anästhesiologie, Universität Ulm, Ulm, Deutschland

${ }^{3}$ Klinik für Anästhesiologie mit Schwerpunkt operative Intensivmedizin, Charité Universitätsmedizin, Humboldt-Universität zu Berlin, Berlin, Deutschland

${ }^{4}$ Klinik für Forensische Psychiatrie, Städtisches Klinikum St. Georg, Leipzig, Deutschland

${ }^{5}$ Tierärztliche Praxis für Neurologie und integrative Schmerzmedizin (iuvet), Pinneberg, Deutschland

${ }^{6}$ Praxis für Rolfing ${ }^{\circledR}$ und Osteopathie, Ravensburg und Ulm, Deutschland

${ }^{7}$ Institut für angewandte Qualitätsförderung und Forschung im Gesundheitswesen (aQua-Institut), Göttingen, Deutschland

${ }^{8}$ Institut für Sportwissenschaften, Arbeitsbereich Sportmedizin und Leistungsphysiologie, Goethe-Universität Frankfurt, Frankfurt am Main, Deutschland

${ }^{9}$ Weimar, Deutschland

${ }^{10}$ Harvard T. H. Chan School of Public Health (Principles and Practice of Clinical Research-Program), Boston, USA

${ }^{11}$ Klinik für Diagnostische und Interventionelle Radiologie, Universitätsklinikum Ulm, Ulm, Deutschland

${ }^{12}$ Orthopädie und Schmerztherapie am Grünen Turm, Ravensburg, Deutschland

${ }^{13}$ Maastricht, Niederlande

${ }^{14}$ Anästhesiologie und Intensivmedizin, Universität Ulm, Ulm, Deutschland

${ }^{15}$ Queensland University of Technology, Brisbane, Australien

\title{
Erratum zu: Akupunktur im Licht der Interdisziplinarität - Erster bis dritter Teil einer Trilogie
}

\section{Erratum zu:}

Dtsch Z Akupunkt (2021) 64:252-259

https://doi.org/10.1007/s42212-021-00412-6

Erratum zu:

Dtsch Z Akupunkt (2021) 64:194-200

https://doi.org/10.1007/s42212-021-00394-5

\section{Erratum zu:}

Dtsch Z Akupunkt (2021) 64:113-119

https://doi.org/10.1007/s42212-021-00382-9

Der Autor Jaap van der Wal war bis 2012 als Dozent Anatomie und Embryologie an der Universität Maastricht tätig.

Die vorliegenden Artikel sind nach seiner Pensionierung entstanden und reflektieren nicht das wissenschaftliche Werk der Abteilung Anatomie und Embryologie dieser Universität.

Wir bitten um Beachtung der aktualisierten Autorenadresse.

\section{Korrespondenzadresse}

\section{Katharina Helbig}

Klinik für Anästhesie, Intensivmedizin, Notfallmedizin und Schmerztherapie,

SRH Kliniken Landkreis Sigmaringen $\mathrm{GmbH}$ Hohenzollernstr. 40, 72488 Sigmaringen, Deutschland helbig.anaesthesie@web.de

Die Online-Versionen der Originalartikel sind unter https://doi.org/10.1007/s42212-021-00412-6, https://doi.org/10.1007/s42212-021-00394-5, https://doi.org/10.1007/s42212-021-00382-9 zu finden.

Deutsche Zeitschrift für Akupunktur 2022 • 65 (1): 4 https://doi.org/10.1007/s42212-021-00440-2

Angenommen: 17. Dezember 2021

Online publiziert: 20. Januar 2022

(c) The Author(s), under exclusive licence to Springer Medizin Verlag

GmbH, ein Teil von Springer Nature 2022 